\title{
Comparison of fractional flow reserve of composite Y-grafts with saphenous vein or right internal thoracic arteries
}

\author{
David Glineur, MD, Munir Boodhwani, MD, Alain Poncelet, MD, Laurent De Kerchove, MD, \\ Pierre Yves Etienne, MD, Philippe Noirhomme, MD, Paul Deceuninck, MD, Xavier Michel, MD, \\ Gebrine El Khoury, MD, and Claude Hanet, MD, PhD
}

\begin{abstract}
Background: Composite Y-grafts, using the left internal thoracic artery as the inflow, allow a more efficient use of conduits without the need to touch a diseased ascending aorta. Among other conduits, the saphenous vein graft may be an alternative to the radial artery in elderly patients.
\end{abstract}

\begin{abstract}
Patients and Methods: We evaluated the hemodynamic characteristics of 17 composite Y-grafts made with the left internal thoracic artery anastomosed to the left anterior descending coronary artery in all cases and with either the free right internal thoracic artery (RITA group, $\mathrm{n}=10$ ) or a saphenous vein graft (SVG group, $\mathrm{n}=7$ ) implanted proximally to the left internal thoracic artery and distally to the circumflex territory 6 months after the operation.
\end{abstract}

\begin{abstract}
Results: At baseline, the pressure gradient measured with a 0.014-inch pressure wire was minimal between the aorta and the internal thoracic artery stem $(2 \pm 1 \mathrm{~mm} \mathrm{Hg})$, the internal thoracic artery and left anterior descending $(4 \pm 2 \mathrm{~mm} \mathrm{Hg})$, the internal thoracic artery and left circumflex $(3 \pm 1 \mathrm{~mm} \mathrm{Hg})$, and the saphenous vein graft and left circumflex $(2 \pm 2 \mathrm{~mm} \mathrm{Hg})$. During hyperemia induced by adenosine, the pressure gradient increased significantly to $6 \pm 2 \mathrm{~mm} \mathrm{Hg}$ in the internal thoracic artery stem, $9 \pm 4 \mathrm{~mm} \mathrm{Hg}$ in the internal thoracic artery and left anterior descending artery, $9 \pm 3 \mathrm{~mm} \mathrm{Hg}$ in the internal thoracic artery and left circumflex, and $7 \pm 4 \mathrm{~mm} \mathrm{Hg}$ in the saphenous vein graft and left circumflex. Fractional flow reserve was $0.94 \pm 0.02$ in internal thoracic artery stem, $0.90 \pm 0.04 \mathrm{~mm} \mathrm{Hg}$ in the internal thoracic artery and left anterior descending, $0.91 \pm 0.03 \mathrm{~mm} \mathrm{Hg}$ in the internal thoracic artery and left circumflex, and $0.92 \pm 0.06 \mathrm{~mm} \mathrm{Hg}$ in the saphenous vein graft and left circumflex. No difference between the two types of composite Y-grafts was observed for pressure gradients or fractional flow reserve measured in internal thoracic artery stem or in distal branches.
\end{abstract}

Conclusions: Composite Y-grafts with saphenous vein or right internal thoracic arteries allow similar and adequate reperfusion of the left system with minimal resistance to maximal flow and an even distribution of flow in both distal branches. (J Thorac Cardiovasc Surg 2010;140:639-45)

Composite Y-grafts allow a more efficient use of graft conduits and have been widely used since their description by Barra, ${ }^{1}$ Tector, ${ }^{2}$ and their associates. Theoretical benefits of a composite Y-graft configuration, using the left internal thoracic artery (LITA) as the inflow, are the absence of aortic manipulation during off-pump surgery and therefore a reduced risk of postoperative neurologic complications. ${ }^{3,4}$ Furthermore, this graft configuration can provide the possibility of minimally invasive multivessel coronary artery bypass through a small left thoracotomy. However, important concerns exist regarding this graft configuration, including

\footnotetext{
From the Department of Cardiovascular Medicine and Surgery, University of Louvain Medical School, Brussels, Belgium.

This study was supported by grant No. 3.4600 .04 from the Fonds de la Recherche Scientifique Médicale, Brussels, Belgium.

Disclosures: None.

Received for publication April 28, 2009; revisions received Sept 24, 2009; accepted for publication Nov 6, 2009; available ahead of print Feb 18, 2010.

Address for reprints: David Glineur, MD, Service de Chirurgie cardiovasculaire et

thoracique, Cliniques Universitaires Saint-Luc-U.C.L.90, Avenue Hippocrate

10/6107, 1200 Bruxelles, Belgium (E-mail: david.glineur@uclouvain.be).

$0022-5223 / \$ 36.00$

Copyright (C) 2010 by The American Association for Thoracic Surgery

doi:10.1016/j.jtcvs.2009.11.013
}

(1) the potential steal phenomenon of the LITA by the second graft, (2) capacity of this graft configuration to revascularize completely the coronary system, and (3) potential flow competition with the native coronary artery. ${ }^{5} \mathrm{We}^{6}$ previously demonstrated that a Y-graft configuration using both internal thoracic arteries (ITAs) allows an adequate revascularization of the whole left coronary system. The resistance is similar in both branches of the Y-graft, which excludes the possibility of a steal from one to the other branch during periods of high myocardial blood flow demand. $\mathrm{We}^{7}$ also showed that the function of the right ITA (RITA) used as a Y-graft was significantly improved when used on several branches of the circumflex artery or on a severely narrowed first circumflex. Grafting of the intermediate branch or of a right coronary artery (RCA) has a negative prognostic influence on graft function.

In elderly patients ( $>75$ years) the choice of the second conduit complementary to the LITA has been debated. Indeed, the use of two ITAs instead of one has a significant benefit in terms of survival or freedom from major adverse cardiocerebro event ${ }^{8}$ only after 10 years. For this reason, among other conduits, the saphenous vein graft (SVG) 


$$
\begin{aligned}
& \text { Abbreviations and Acronyms } \\
& \text { FFR }=\text { fractional flow reserve } \\
& \text { ITA }=\text { internal thoracic artery } \\
& \text { LITA }=\text { left internal thoracic artery } \\
& \text { LAD = left anterior descending } \\
& \text { OB }=\text { obtuse marginal branch of left circumflex } \\
& \text { artery } \\
& \text { RCA }= \text { right coronary artery } \\
& \text { RITA }= \text { right internal thoracic artery } \\
& \text { SVG }= \text { saphenous vein graft }
\end{aligned}
$$

may be an alternative to the radial artery in these elderly patients. Moreover, in a recent prospective randomized trial, Hayward and colleagues ${ }^{9}$ have not found any clinical benefit of the radial artery over the SVG at 6 years' follow-up.

Previous reports have described the implantation of the SVG into the LITA because of a diseased aorta with different conflicting results. ${ }^{10-12}$ The potential disadvantages of such composite arteriovenous configurations are their dependence on the flow capacity of the proximal LITA and the risk of an uneven distribution of flow in both branches or a malperfusion of one myocardial territory. ${ }^{13,14}$

The present study was designed to evaluate the difference of conductance of arterial Y-graft configurations versus the arteriovenous $\mathrm{Y}$-graft by directly measuring the perfusion pressure in the main stem of the pedicled LITA and in each of the $\mathrm{Y}$ assembling limbs.

\section{METHODS \\ Patients}

Between 2003 and 2007, 210 patients underwent nonemergeny coronary artery bypass surgery using the LITA to the left anterior descending artery (LAD) and the RITA to the circumflex territory in a composite Y-graft configuration. Of these, 146 patients consented to a follow-up angiographic study. Criteria for inclusion in the fractional flow reserve (FFR) study protocol were the absence of major tortuosities of the graft, patency of all anastomoses with a good runoff flow to the grafted arterial segments, and absence of severe wall motion abnormality in the perfusion zone. Exclusion criteria were renal insufficiency, peripheral vascular disease, and history of neurologic events. Of the 146 patients undergoing follow-up angiography, 10 patients benefited from a more complete examination with FFR measurement (RITA group).

During the same period of time, 35 patients underwent the same procedure but using the LITA and the SVG implanted into the LITA. The SVG was used in these patients rather than a second ITA because of the presence of uncontrolled diabetes, severe obstructive pulmonary disease, advanced age ( $>75$ years), and/or dialysis-dependent renal failure. Of these, 7 fulfilled the selection criteria and consented to follow-up angiography with FFR measurement (SVG group).

All 17 patients had off-pump operations. When doing an off-pump procedure, we aim to avoid aortic manipulation and thus prefer using a Y-graft configuration and sequential anastomoses. In all cases, the LITA was used to revascularize the LAD territory (mean anastomoses: RITA group, $1.2 \pm$ 0.4 ; SVG group, $1.6 \pm 0.5 ; P=$ not significant) and the free graft was anastomosed proximally to the LITA and distally to the left circumflex (obtuse marginal [OB]) territory (mean anastomoses: RITA group, $1.9 \pm 0.6$; SVG
TABLE 1. Patient characteristics

\begin{tabular}{lcc}
\hline & RITA group I & SVG group II \\
\hline Mean age (y) & 59 & 71 \\
Male gender (\%) & 70 & 100 \\
Family history (\%) & 20 & 42 \\
Arterial hypertension (\%) & 80 & 57 \\
Hyperlipidemia (\%) & 90 & 29 \\
Diabetes (\%) & 20 & 42 \\
\hline
\end{tabular}

RITA, Right internal thoracic artery; $S V G$, saphenous vein graft.

group, $2 \pm 0.6 ; P=$ not significant). Patient characteristics and operative data are shown in Table 1. Data on preoperative coronary stenoses are shown in Table 2. There were no significant differences between groups in the preoperative coronary lesions except for the OM2 lesion, which was more severe in the RITA group $(P=.02)$.

The study protocol was approved by the ethics committee of our institution. All patients gave informed consent at the time of bypass surgery and before the angiographic investigation and completed the study protocol without complication.

\section{Surgical Technique}

All patients were operated on by median sternotomy, without cardiopulmonary bypass. The pedicled ITA was harvested together with the surrounding veins, muscle, and fascia. The cautery was always used and the side branches were clipped. The RITA was connected to the pedicled LITA with end-to-side anastomosis. The Y-graft was constructed before the distal bypass at the beginning of the procedure was done.

The in situ LITA was prepared at the chosen site and then clamped. A longitudinal incision was performed, 6 to $8 \mathrm{~mm}$ long. The free graft (RITA or SVG) was prepared, opened obliquely at its tip, and then extended to the graft. A running stitch with an 8-0 polypropylene suture (Prolene; Ethicon, Inc, Somerville, NJ), starting from the heel of the free graft, was used. The in situ ITA was then unclamped and the proximal anastomosis was carefully checked for any bleeding.

\section{Study Protocol}

Patients underwent cardiac catheterization by the standard femoral approach. To avoid any vasoactive drug interference, all patients interrupted all vasoactive medications 24 hours before the systematic 6-month angiographic study. Selective catheterization of the ostium of the LITA was achieved by using a $5 \mathrm{~F}$ guiding catheter without side holes. This catheter was connected to a fluid-filled pressure transducer zeroed at the mid-chest level. After intragraft administration of $1 \mathrm{mg}$ of isosorbide dinitrate, a biplane angiogram of the graft was obtained. A 0.014-inch electronic sensor-tipped wire (PressureWire; Radi Medical Systems, Uppsala, Sweden) was advanced to the tip of the guiding catheter to ensure that the pressures recorded in the ostium of the graft through the fluid-filled catheter and the pressure wire were identical. The wire was then advanced in the distal part of the LITA main stem, close to the proximal anastomosis of the free graft (ITA-stem). The pressures were simultaneously measured in the ostium of the graft and in the ITA-stem under basal conditions. Coronary arteriolar vasodilation was then induced by intragraft bolus injection of $40 \mu \mathrm{g}$ of adenosine to produce transient maximal hyperemia as previously described. ${ }^{7,15,16}$ Pressure signals were recorded continuously until distal pressure returned to baseline values.

The pressure wire was then successively advanced in each of the two distal branches, the pressure sensor being carefully positioned within the last centimeter preceding the first distal anastomosis on the LAD (ITA-LAD) or left circumflex (ITA-OM or SVG-OM) system (Figure 1). At each measurement point, the same pressure measurements were repeated in baseline conditions and during a maximal hyperemia induced by bolus injections of adenosine. When the pressure sensor was pulled back in the guiding catheter, pressures were checked to exclude any drift of the transducers. 
TABLE 2. Second graft and preoperative coronary stenosis

\begin{tabular}{|c|c|c|c|c|c|c|}
\hline $\begin{array}{c}\text { Patient } \\
\text { No. } \\
\end{array}$ & $\begin{array}{c}\text { Second } \\
\text { graft }\end{array}$ & $\begin{array}{c}\text { LAD: } \% \\
\text { stenosis }\left(\mathbf{m m}^{*}\right)\end{array}$ & $\begin{array}{c}\text { DG: } \% \\
\text { stenosis }\left(\mathbf{m m}^{*}\right)\end{array}$ & $\begin{array}{c}\text { OM1: } \% \\
\text { stenosis }\left(\mathrm{mm}^{*}\right)\end{array}$ & $\begin{array}{c}\text { OM2: } \% \\
\text { stenosis }\left(\mathrm{mm}^{*}\right)\end{array}$ & $\begin{array}{c}\text { PDA: } \% \\
\text { stenosis }\left(\mathrm{mm}^{*}\right) \\
\end{array}$ \\
\hline 1 & RITA & $45(1.3)$ & & 100 & 100 & \\
\hline 2 & RITA & 100 & & $70(0.8)$ & $65(0.9)$ & \\
\hline 3 & RITA & $85(0.7)$ & & $63(1.2)$ & $57(0.58)$ & $53(0.7)$ \\
\hline 4 & RITA & $85(0.7)$ & $63(1.2)$ & $57(0.58)$ & 53 (1.16) & \\
\hline 5 & RITA & $63(1.1)$ & $70(1)$ & $65(1.2)$ & $70(0.7)$ & \\
\hline 6 & RITA & $70(1.13)$ & & $60(1.2)$ & $51(1.7)$ & \\
\hline 7 & RITA & 100 & & $56(1.14)$ & 100 & \\
\hline 8 & RITA & $50(1.3)$ & & (49 1.54) & $75(0.65)$ & \\
\hline 9 & RITA & $67(0.7)$ & & $50(1)$ & $60(0.8)$ & \\
\hline 10 & RITA & $66(0.6)$ & & $40(1.16)$ & 100 & \\
\hline \multicolumn{2}{|c|}{ Mean $\%$ stenosis $\left(\mathrm{mm}^{*}\right)$} & $73(0.9)$ & $67(1.1)$ & $61(1.1)$ & $73(0.9)$ & $53(0.7)$ \\
\hline 11 & SVG & $59(0.81)$ & $60(0.81)$ & $63(0.9)$ & & 100 \\
\hline 12 & SVG & $70(0.5)$ & $65(0.8)$ & $60(0.7)$ & $49(0.4)$ & 100 \\
\hline 13 & SVG & 100 & & $51(0.84)$ & 47 (1) & \\
\hline 14 & SVG & $46(1.37)$ & & $44(1.45)$ & & 100 \\
\hline 15 & SVG & $55(1.23)$ & $60(0.8)$ & $58(1.28)$ & & 100 \\
\hline 16 & SVG & $70(0.9)$ & & $70(0.8)$ & $62(0.9)$ & 100 \\
\hline 17 & SVG & $65(0.84)$ & & $69(0.96)$ & $57(0.7)$ & 100 \\
\hline \multicolumn{2}{|c|}{ Mean $\%$ stenosis $\left(\mathrm{mm}^{*}\right)$} & $66(1.1)$ & $52(0.8)$ & 59 (1) & $54(0.8)$ & 100 \\
\hline
\end{tabular}

$L A D$, Left anterior descending coronary artery; $D G$, first diagonal branch of the left anterior descending coronary artery; OM1 and OM2, first and second marginal branches of the left circumflex coronary artery; $P D A$, posterior descending artery; RITA, right internal thoracic artery; SVG, saphenous vein graft. * Minimum lumen diameter in millimeters.

\section{Pressure Data Analysis}

Pressures recorded at different measurement points were compared under basal conditions and during maximal hyperemia. The gradient in mean intragraft pressure between the ostium of the graft and each of the three distal measurement points is expressed in absolute values. From pressure measurements obtained during maximal hyperemia, FFR was computed as an index of resistance to blood flow of the different segments of the graft. FFR is calculated as the ratio of distal pressure $\left(\mathrm{P}_{\mathrm{d}}\right)$ divided by proximal intragraft $\left(\mathrm{P}_{\mathrm{p}}\right)$ pressure as follows: FFR $=\mathrm{P}_{\mathrm{d}} / \mathrm{P}_{\mathrm{p}}$. ${ }^{7}$ In the absence of resistance along a vascular segment, there is no pressure decline and FFR equals unity. The larger the resistance to blood flow of a graft segment, the greater the drop in perfusion pressure across this segment and thus the smaller the FFR.

\section{Angiographic Analysis}

Preoperative angiograms were analyzed using the CAAS II system (Pie Medical, Maastricht, The Netherlands) as previously described. ${ }^{17}$ The minimal lumen diameter and percent diameter stenosis of the native diseased coronary were measured in two near orthogonal projections and the mean value of these measurements was considered for statistical analyses. Graft sizes were measured on the postoperative angiograms at the 3 pressure measurement points under basal conditions: in the ITA-stem and in each of the two distal branches of the composite Y graft assembling.

\section{Statistical Analysis}

All data are expressed as mean \pm SD. Pressure differences between the ostium and distal measurement points of the graft at baseline and during hyperemia and FFR measured at different points in the same grafts were compared by paired Student $t$ tests. Adjustment for multiple comparisons.

\section{RESULTS}

Preoperative angiograms showed no significant differences for the percentage of stenosis between the 2 groups:
LAD: RITA group, $68 \% \pm 16 \%$, versus $\mathrm{SVG}$ group, $67 \% \pm 19 \%(P=.8)$; OM1: RITA group, $60 \% \pm 16 \%$, versus $\mathrm{SVG}$ group, $55 \% \pm 8 \%(P=.6)$; OM2: RITA group, $75 \% \pm 18 \%$, versus $\mathrm{SVG}$ group, $55 \% \pm 20 \%$ $(P=.02)$ (Table 2).

In each patient, the baseline postoperative angiogram showed a patent composite Y-graft with a complete opacification of all target coronary segments. Selective positioning of the pressure sensor in the ITA-stem and in both distal branches was possible in all.

In basal conditions, a small pressure gradient was measured between the ostium of the graft and each of the three measurement points (Table 3). Comparisons of basal conditions of the pressure gradient between the main stem and the distal branches were as follows: in the RITA group, main stem $(1.7 \pm 1.5 \mathrm{~mm} \mathrm{Hg})$ versus ITA-LAD $(3.4 \pm 2.9 \mathrm{~mm}$ $\mathrm{Hg} ; P=.028)$ versus ITA-OM $(2.7 \pm 1.5 \mathrm{~mm} \mathrm{Hg} ; P=$ $.12)$; in the $\mathrm{SVG}$ group, main stem $(1.3 \pm 1.2 \mathrm{~mm} \mathrm{Hg})$ versus ITA-LAD $(4.1 \pm 1.6 \mathrm{~mm} \mathrm{Hg} ; P=.0029)$ versus $\mathrm{SVG}-\mathrm{OM}$ $(2.3 \pm 1.7 \mathrm{~mm} \mathrm{Hg} ; P=.29)$. This shows that the drop of pressure is significantly more important in the distal LAD branch then in the OM branch in both groups.

Adenosine injection resulted in a consistent increase in pressure gradient in every patient at each of the measurement points (Table 3). Comparison of gradient in hyperemia between the main stem and distal branches in the RITA group was as follows: main stem $(6.2 \pm 2.3 \mathrm{~mm} \mathrm{Hg})$ versus ITA-LAD $(9.3 \pm 4.7 \mathrm{~mm} \mathrm{Hg} ; P=.03)$ versus ITA-OM 

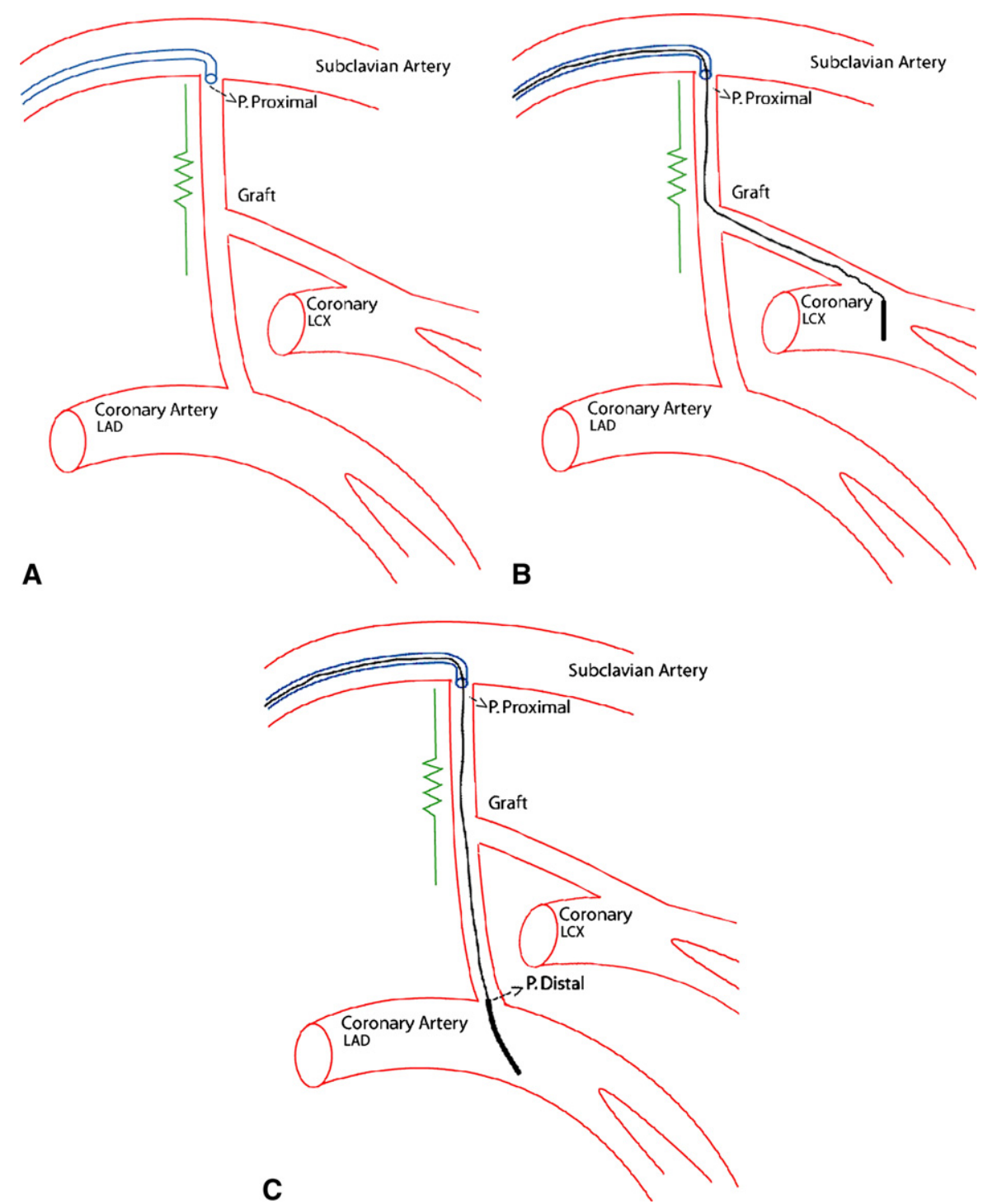

FIGURE 1. Schematic representation of pressure measurement sites. Measurement in the main stem (A) in the LCX graft (B), and in the distal LITA-LAD anastomosis (C). $L C X$, Left circumflex; $L A D$, left anterior descending coronary artery.

$(8.8 \pm 2.6 \mathrm{~mm} \mathrm{Hg} ; P=.003)$; in SVG group, comparisons were as follows: main stem $(5.1 \pm 1.9 \mathrm{~mm} \mathrm{Hg})$ versus ITA-LAD $(9.4 \pm 2.1 \mathrm{~mm} \mathrm{Hg} ; P=.01)$ versus SVG-OM $(7.3 \pm 4.2 \mathrm{~mm} \mathrm{Hg} ; P=.15)$. This shows that in hyperemia induced by adenosine, the pressure drop increased significantly in both distal branches of the Y-graft in the RITA group, whereas in the SVG group the pressure drop in the OM branch was not statistically significant.

Comparison of the distal branch pressure in hyperemia was not significantly different between the 2 groups: graftOM branch $(P=.42)$ and ITA-LAD $(P=.94)$ (Table 3$)$.

Comparison of the pressure gradient between the ostium and each branch of the Y-graft in every individual patient showed an even distribution of perfusion pressure between both branches of the graft, the difference in pressure drop be- tween ITA-LAD and graft-OM remaining within a range of $5 \mathrm{~mm} \mathrm{Hg}$ during maximal hyperemia.

FFR in the ITA-stem averaged $0.94 \pm 0.03$ in the RITA group and $0.94 \pm 0.02$ in the SVG group $(P=.77)$, reflecting a minimal resistance to flow during maximal hyperemia induced by adenosine. Comparison of distal FFR between the 2 groups showed no significant differences (Table 3).Comparison of FFR between the different measurements sites is shown in Figure 2.

Graft size measurement (Table 3) showed no significant differences for the ITA main stem between the 2 groups (RITA group, $3.1 \pm 0.3 \mathrm{~mm}$, vs SVG group, $3.0 \pm 0.3$ $\mathrm{mm} ; P=.52$ ) and a significant difference for the distal branches of the Y-grafts (LAD: RITA group, $2.5 \pm 0.3$ $\mathrm{mm}$, versus SVG group, $2.2 \pm 0.1 \mathrm{~mm} ; P=.04)$; OM: 
TABLE 3. Graft pressures and dimensions

\begin{tabular}{lccc}
\hline & RITA group & SVG group & $\boldsymbol{P}$ values \\
\hline ITA-stem & & & \\
$\quad$ Basal (mean gradient) & $1.7 \pm 1.5$ & $1.3 \pm 1.2$ & .55 \\
Adenosine (mean gradient) & $6.2 \pm 2.3$ & $5.1 \pm 1.9$ & .32 \\
FFR & $0.94 \pm 0.03$ & $0.94 \pm 0.02$ & .77 \\
Graft size (basal condition) & $3.1 \pm 0.3$ & $3 \pm 0.3$ & .52 \\
ITA-LAD & & & \\
Basal (mean gradient) & $3.4 \pm 2.9$ & $4.1 \pm 1.6$ & .51 \\
Adenosine (mean gradient) & $9.3 \pm 4.7$ & $9.4 \pm 2.1$ & .94 \\
FFR & $0.91 \pm 0.04$ & $0.88 \pm 0.04$ & .16 \\
Graft size (basal condition) & $2.5 \pm 0.3$ & $2.2 \pm 0.1$ & .04 \\
Graft- OM & & & \\
Basal (mean gradient) & $2.7 \pm 1.5$ & $2.3 \pm 1.7$ & .61 \\
Adenosine (mean gradient) & $8.8 \pm 2.6$ & $7.3 \pm 4.2$ & .42 \\
FFR & $0.91 \pm 0.03$ & $0.92 \pm 0.06$ & .94 \\
Graft size (basal condition) & $2.4 \pm 0.5$ & $3.2 \pm 0.2$ & .001 \\
\hline
\end{tabular}

FFR, Fractional flow reserve; $O M$, obtuse marginal branches of the left circumflex coronary artery; $L A D$, left anterior descending coronary artery; ITA, internal thoracic artery.

RITA group, $2.4 \pm 0.5 \mathrm{~mm}$, versus $\mathrm{SVG}$ group, $3.2 \pm 0.2$ $\mathrm{mm} ; P=.001)$. In other words, the LITA-LAD portion was smaller in the RITA group and the SVG was significantly larger than the RITA.

\section{DISCUSSION}

This study shows that a Y-graft configuration with a free RITA or an SVG attached to a pedicled left ITA allows an adequate revascularization of the whole coronary system with an even distribution of perfusion pressure in both distal branches and a minimal resistance to maximal blood flow. The resulting gradual decrease in pressure along the graft is negligible in basal conditions and remains small during a maximal hyperemia induced by coronary arteriolar vasodilation. Not surprisingly, most of the pressure drop observed during hyperemia occurs across the common limb of the Ygraft where blood flow is maximal. As expected, the SVG is larger than the RITA to the OM.

Composite Y-grafts, using the LITA as the inflow, allow a more efficient use of conduits. Indeed, this graft configuration allows performing complete myocardial revascularization with 2 grafts only, which would not be possible if the RITA was used in situ. Another advantage of this configuration is the absence of aortic manipulation and the resulting neurologic benefits. Kotoh and colleagues ${ }^{18}$ have identified partial aortic clamping as an independent factor strongly associated with cerebral infarction after off-pump coronary artery bypass grafting. Kapetanakis and associates ${ }^{3}$ have also found that aortic manipulation during bypass grafting is a contributing mechanism for postoperative stroke. The incidence of postoperative stroke increases with increased levels of aortic manipulation. This is particularly true in elderly patients considering the high prevalance of moderate-to-severe atherosclerotic aortic disease. These patients are particularly prone to stroke-related embolization after aortic manipula-
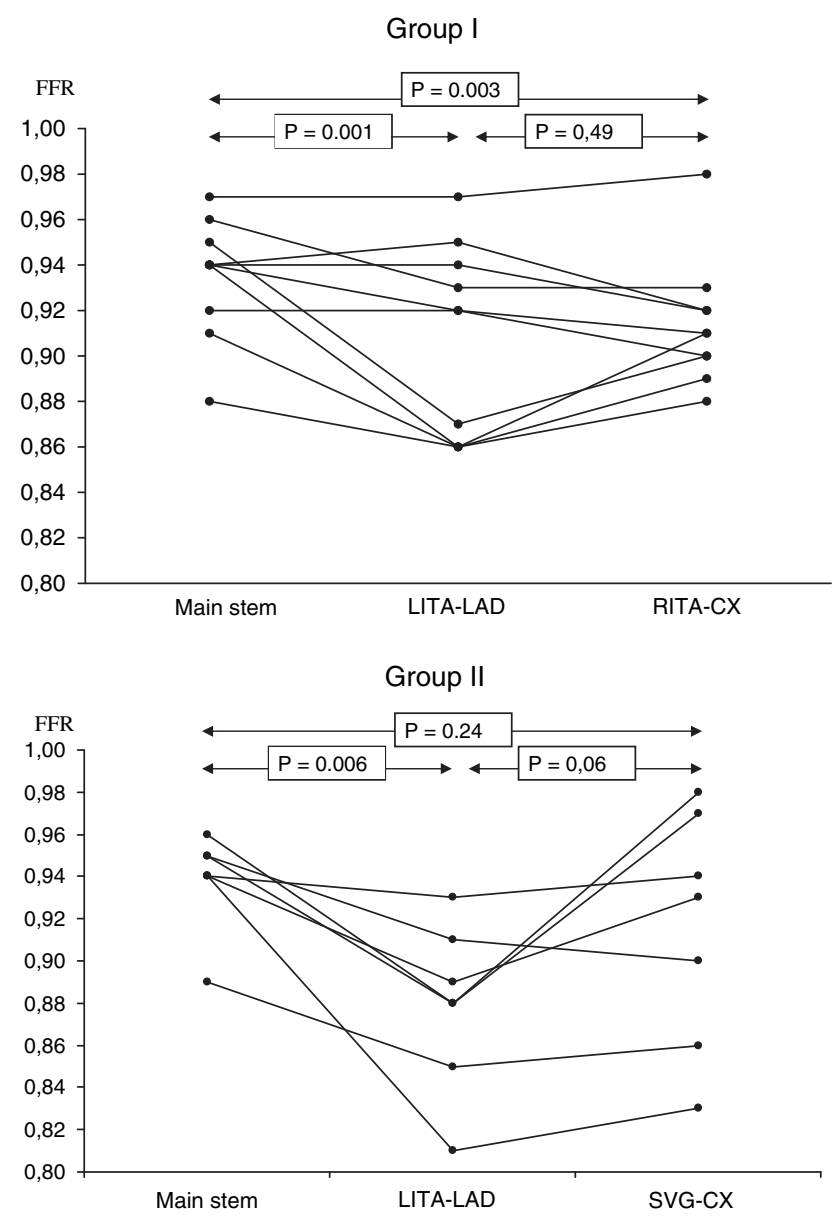

FIGURE 2. Individual values of fractional flow reserve $(F F R)$ measured at different points of the Y-graft: ITA-stem, In the main part of the graft immediately before the implantation of the free graft on the LITA; ITA-LAD, within the last centimeter preceding the first distal anastomosis of the LITA on the LAD vascular bed and graft-LCX within the last centimeter preceding the first distal anastomosis of the free graft on the LCX system. $I T A$, Internal thoracic artery; $L I T A$, left internal thoracic artery; $L A D$, left anterior descending coronary artery; RITA, right internal thoracic artery; $C X$, circumflex artery; $S V G$, saphenous vein graft.

tions. ${ }^{19}$ For this reason, Oren Lev-Ran and colleagues ${ }^{4}$ compared 103 patients operated on without aortic manipulation (clampless group) with 57 patients having off-pump coronary artery bypass using partial aortic clamps during the construction of proximal aortic anastomoses (side-clamp group). They concluded that clampless off-pump coronary bypass in elderly patients attenuates the risk of major neurologic complications irrespective of the aortic screening strategy.

Moreover, Y-graft configuration is a safe and reproducible technique. $\mathrm{We}^{20}$ have recently published the results of a randomized comparison between in situ bilateral ITA versus bilateral ITA Y-grafting, showing that there were no significant clinical or angiographic differences at 20 months of follow-up.

Nevertheless, a major problem for the Y-graft configuration is the possibility of flow competition. Pevni and 
colleagues ${ }^{5}$ analyzed 203 postoperative coronary angiograms in 163 patients who underwent bilateral ITA grafting using the composite T-graft technique. They concluded that the composite T-graft technique of bilateral ITA grafting should be reserved for patients with severe $(\geq 70 \%)$ LAD and circumflex arterial stenosis because of the possible flow competition with the native coronary circulation.

Previous studies have described the use of the SVG in a composite manner with some conflicting results. Gaudino and associates ${ }^{10}$ studied 25 patients in whom the SVG was implanted into the LITA. They found that the short-term patency of composite Y ITA-SVG was suboptimal and markedly influenced by distal runoff and native flow competition. In fact, all cases of ITA malfunction were reported in the part of the ITA distal to the SVG anastomosis and always in patients in whom the artery was used to revascularize a coronary artery with less than $70 \%$ stenosis $(P=0.02)$. We did not find this phenomenon in our series, in which all distal ITA grafts to the LAD were patent and with a normal diameter.

The concept of pressure-derived FFR has been developed to assess the physiologic significance of coronary stenosis. ${ }^{16,21}$ FFR is calculated as the ratio of distal coronary pressure divided by aortic pressure during maximal hyperemia. In the absence of resistance along an artery, there is no pressure decline and FFR equals unity. This index is unaffected by conditions known to influence baseline myocardial flow and takes into account the contribution of the collateral blood supply to maximal myocardial perfusion, ${ }^{21}$ which is particularly relevant after bypass surgery. FFR is considered specific to epicardial conductance and has been proposed as a clinical tool to identify stenoses capable of inducing reversible ischemia. Previous studies have shown that an FFR value less than 0.75 discriminates functionally significant coronary lesions that justify revascularization. $^{22}$

As with epicardial coronary arteries, one expects a bypass graft to allow perfusion of the myocardial coronary territory with a minimal decrease in perfusion pressure and an FFR close to unity. The FFR measured in the distal branches of the Y-graft in our patients are close to those reported in the distal part of "normal" coronary arteries of patients with a remote coronary lesion ${ }^{21}$ and reflect some minimal resistance to blood flow along the graft. All values of FFR were superior to 0.75 , the cutoff for inducible ischemia.

\section{STUDY LIMITATIONS}

The main limitation of this study is its retrospective nature, with a small sample size.

\section{CONCLUSION}

Composite Y-grafts using the saphenous vein or the RITA allow similar and adequate reperfusion of the left system with minimal resistance to maximal flow and an even distribution of flow in both distal branches. SVG implantation into the LITA in elderly patients or patients with a diseased aorta could be a useful alternative to conventional revascularization strategies.

\section{References}

1. Barra JA, Mondine P, Mahlab A, Bezon E, Rukbi I, Slimane AK, et al. Right internal mammary artery reimplantation into the left internal mammary artery: Y anastomosis. 25 cases. Ann Chir. 1991;45:661-6.

2. Tector AJ, Amundsen S, Schmahl TM, Kress DC, Peter M. Total revascularization with T grafts. Ann Thorac Surg. 1994;57:8-9.

3. Kapetanakis EI, Stamou SC, Dullum MK, Hill PC, Haile E, Boyce SW, et al. The impact of aortic manipulation on neurologic outcomes after coronary artery bypass surgery: a risk-adjusted study. Ann Thorac Surg. 2004;78:1564-71.

4. Lev-Ran O, Braunstein R, Sharony R, Kramer A, Paz Y, Mohr R, et al. No-touch aorta off-pump coronary surgery: the effect on stroke. J Thorac Cardiovasc Surg. 2005;129:307-13.

5. Pevni D, Hertz I, Medalion B, Kramer A, Paz Y, Uretzky G, et al. Angiographic evidence for reduced graft patency due to competitive flow in composite arterial T-grafts. J Thorac Cardiovasc Surg. 2007;133:1220-5.

6. Glineur D, Hanet C, D'hoore W, Poncelet A, De Kerchove L, Etienne PY, et al. Causes of non-functioning right internal mammary used in a Y-graft configuration: insight from a 6-month systematic angiographic trial. Eur J Cardiothorac Surg. 2009;36:129-35; discussion 135-136.

7. Glineur D, Noirhomme P, Reisch J, El Khoury G, Astarci P, Hanet C. Resistance to flow of arterial Y-grafts 6 months after coronary artery bypass surgery. Circulation. 2005;112(9 Suppl):I281-5.

8. Rankin JS, Tuttle RH, Wechsler AS, Teichmann TL, Glower DD, Califf RM. Techniques and benefits of multiple internal mammary artery bypass at 20 years of follow-up. Ann Thorac Surg. 2007;83:1008-14.

9. Hayward PA, Hare DL, Gordon I, Buxton BF. Effect of radial artery or saphenous vein conduit for the second graft on 6-year clinical outcome after coronary artery bypass grafting. Results of a randomised trial. Eur J Cardiothorac Surg. 2008;34: $113-7$.

10. Gaudino M, Alessandrini F, Pragliola C, Luciani N, Trani C, Burzotta F, et al. Composite $\mathrm{Y}$ internal thoracic artery-saphenous vein grafts: short-term angiographic results and vasoreactive profile. J Thorac Cardiovasc Surg. 2004;127: 1139-44.

11. Murphy DA, Hatcher CR Jr. Coronary revascularization in the presence of ascending aortic calcification: use of an internal mammary artery-saphenous vein composite graft. J Thorac Cardiovasc Surg. 1984;87:789-91.

12. Brodman R, Robinson G. Internal mammary artery-saphenous vein composite conduit: an alternative for the proximal coronary anastomosis. Ann Thorac Surg. 1981;31:370-2.

13. Wendler O, Hennen B, Markwirth T, König J, Tscholl D, Huang Q, et al. T grafts with the right internal thoracic artery to left internal thoracic artery versus the left thoracic artery and radial artery: flow dynamics in the internal thoracic artery main stem. J Thorac Cardiovasc Surg. 1999;118:841-8.

14. Sakaguchi G, Tadamura E, Ohnaka M, Tambara K, Nishimura K, Komeda M. Composite arterial Y graft has less coronary flow reserve than independent grafts. Ann Thorac Surg. 2002;74:493-6.

15. Glineur D, Poncelet A, El Khoury G, D 'hoore W, Astarci P, Zech F, et al. Fractional flow reserve of pedicled internal thoracic artery and saphenous vein grafts 6 months after bypass surgery. Eur J Cardiothorac Surg. 2007;31: 376-81.

16. De Bruyne B, Hersbach F, Pijls NHJ, Bartunek J, Bech JW, Heyndrickx GR, et al. Abnormal epicardial coronary resistance in patients with diffuse atherosclerosis but "normal"' coronary angiography. Circulation. 2001;104:2401-6.

17. Hanet C, Robert A, Wyns W. Vasomotor response to ergometrine and nitrates of saphenous vein grafts, internal mammary artery grafts and grafted coronary arteries late after bypass surgery. Circulation. 1992;86(5 Suppl):II210-6.

18. Kotoh K, Fukahara K, Doi T, Nagura S, Misaki T. Predictors of early postoperative cerebral infarction after isolated off-pump coronary artery bypass grafting. $T$. Ann Thorac Surg. 2007;83:1679-83.

19. Wareing TH, Davila-Roman VG, Barzilai B, Murphy SF, Kouchoukos NT. Management of severely atherosclerotic ascending aorta during cardiac operations. J Thorac Cardiovasc Surg. 1992;103:453-62.

20. Glineur D, Hanet C, Poncelet A, D'hoore W, Funken JC, Rubay J, et al. Comparison of bilateral internal thoracic artery revascularization using in situ or Y graft configurations: a prospective randomized clinical, functional, and angiographic midterm evaluation. Circulation. 2008;118(14 Suppl):S216-21. 
21. Pijls NHJ, De Bruyne B, Peels K, van der Voort PH, Bonnier HJRM, Bartunek J, et al. Measurement of fractional flow reserve to assess the functional severity of coronary-artery stenoses. N Engl J Med. 1996;334:1703-8.

22. Kern MJ, Lerman A, Bech JW, De Bruyne B, Eeckhout E, Fearon WF, et al., American Heart Association Committee on Diagnostic and Interventional Cardiac
Catheterization, Council on Clinical Cardiology. Physiological assessment of coronary artery disease in the cardiac catheterization laboratory: a scientific statement from the American Heart Association Committee on Diagnostic and Interven tional Cardiac Catheterization, Council on Clinical Cardiology. Circulation. 2006;114:1321-41. 